\title{
Erratum: T duality and Wald entropy formula in the Heterotic Superstring effective action at first-order in $\alpha^{\prime}$
}

\author{
Zachary Elgood and Tomás Ortín \\ Instituto de Física Teórica UAM/CSIC, \\ C/ Nicolás Cabrera, 13-15, C.U. Cantoblanco, Madrid E-28049, Spain \\ E-mail: zachary.elgood@uam.es, tomas.ortin@csic.es
}

ERRATUM TO: JHEP10(2020)097

ARXIV EPRINT: 2005.11272

While the main formulae and results of our paper $T$ duality and Wald entropy formula in the Heterotic Superstring effective action at first-order in $\alpha^{\prime}$ (Zachary Elgood and Tomás Ortín, JHEP 10 (2020) 097 [arXiv:2005.11272]) remain unaltered, we have detected a number of typos that affect some of the intermediate calculations:

1. The - sign in eq. (4.3a) should be $a+$.

2. A term $+\frac{\alpha^{\prime}}{4} \partial_{a} \log k \partial^{a} \varphi^{2}$ is missing in the integrand in eqs. (4.4) and (4.24).

3. In the second line of eq. (4.17), there is a facrtor of 2 missing in front of the $(\log k)^{2}$ term.

4. The line after eq. (4.19) should read "Half of the last term should be integrated by parts $[\ldots] "$.

5. Eq. (4.20) contains some errors and should read

$$
\hat{\omega}^{\mathrm{YM}}{ }_{a b c}=\omega^{\mathrm{YM}}{ }_{a b c}+3 e_{a}{ }^{\mu} e_{b}{ }^{\nu} e_{c}{ }^{\rho}\left[\partial_{[\mu}\left(k A_{\nu \mid} \varphi_{A} A_{\mid \rho]}^{A}\right)+A_{[\mu} \partial_{\nu \mid}\left(k \varphi_{A} A_{\mid \rho]}^{A}\right)+\frac{3}{2} k \varphi_{A} A_{[\mu}^{A} F_{\nu \rho]}\right] .
$$

6. Eq. (4.21) contains some errors and should read

$$
\begin{aligned}
\hat{\omega}^{\mathrm{L}(0)}{ }_{a b c}= & \omega^{\mathrm{L}(0)}{ }_{a b c}+3 e_{a}^{\mu} e_{b}{ }^{\nu} e_{c}{ }^{\rho}\left[\mathcal{D}_{(-)[\mu}^{(0)} K_{\nu}^{(-)}{ }_{\nu} K_{\rho] e}^{(-)}+\partial_{[\mu}\left(k A_{\nu \mid} \varphi^{\hat{e}_{\hat{f}}} A_{\hat{e} \mid \rho]}^{\hat{\hat{e}}}\right)\right. \\
& \left.+A_{[\mu} \partial_{\nu \mid}\left(k \varphi_{\hat{f}}^{\hat{e}} A_{\hat{e} \mid \rho]}^{\hat{\hat{e}}}\right)+\frac{1}{2} k \varphi^{\hat{e}} A_{\hat{e}[\mu}^{\hat{\hat{e}}} F_{\nu \rho]}\right] .
\end{aligned}
$$


7. A factor of 3 is missing in front of the last term in eq. (4.23b).

8. In the last line of eq. (4.25), the two relative - signs should + es.

9. In the second and third lines of eq. (4.26), the last term in each parenthesis sould carry a + sign, instead of a - sign.

10. The last term in each parenthesis the lines $3,4,5$ and 6 of eq. (4.27) should carry a + sign, instead of a - sign.

11. The signs of the first terms in the lines $6,7,9$ and 10 of eq. (4.28) should be reversed.

Open Access. This article is distributed under the terms of the Creative Commons Attribution License (CC-BY 4.0), which permits any use, distribution and reproduction in any medium, provided the original author(s) and source are credited. 